\title{
Respectful Adolescent Care: A Right of Every Adolescent
}

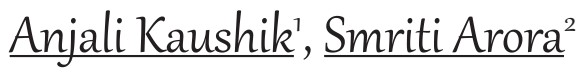 \\ ${ }^{1}$ Tutor, Rufaida College of Nursing, Jamia Hamdard, Delhi, India. \\ ${ }^{2}$ Professor, Amity University Haryana, Haryana, India. \\ DOI: https://doi.org/10.24321/2349.2880.202013
}

\section{I $\quad \mathbf{N} \quad \mathbf{F} \quad \mathbf{O}$}

\section{Corresponding Author:}

Anjali Kaushik, Rufaida College of Nursing, Jamia Hamdard, Delhi, India.

E-mail Id:

anjalikaushik@jamiahamdard.ac.in

Orcid Id:

https://orcid.org/0000-0001-6782-5742

How to cite this article:

Kaushik A, Arora S. Respectful Adolescent Care: A Right of Every Adolescent. Ind J Youth Adol Health 2020; 7(3): 16-19.

Date of Submission: 2020-11-21

Date of Acceptance: 2020-12-18

\section{$\begin{array}{llllllll}\mathbf{A} & \mathbf{B} & \mathbf{S} & \mathbf{T} & \mathbf{R} & \mathbf{A} & \mathbf{C} & \mathbf{T}\end{array}$}

Adolescence is a crucial period in the life of every individual. The significance of adolescence and young adulthood is highlighted in relation to the crucial developmental process of preparing and transitioning to adulthood. During this period, the important developmental milestones are reached, and the young person develops a greater understanding about who they are and form their self-identity as per the Erikson. Adolescents become more independent and autonomous during this time. Adolescence is marked by changes that are not only physical but also psychological, social and emotional. Sometimes due to lack of proper knowledge and guidance, they end up having health related issues like teen pregnancy, STDs and other mental health issues like breakups etc. To tackle all these issues the health professionals should be sensitive and non-judgmental and should provide unbiased care. Adolescents have their own experiences and point of view that should be respected and taken into account. It is important to trust adolescents and their sense of responsibility. Adolescents are often reluctant to visit health facilities. It is therefore important to reach out to them by providing adolescent friendly services. It is their right also to have access to health care whenever they need it. It is essential to have trained and sensitive staff in these health centers so that young people access services in these centers in a confidential and non-judgmental manner.

Keywords: Adolescence, Respectful Adolescent Care, Right, Confidential

\section{Introduction}

India has the largest adolescent population in the world (253 million, Census 2011). Although considered healthy phase of life, adolescents do have several issues requiring medical care. In this age the mortality and morbidity patterns begin to resemble more like adults as accidents, injuries, and violence become important causes of deaths. The adolescents in India suffer from common infectious diseases, nutritional deficiency diseases like anemia (more than half girls and one-third boys have anemia), and also mental health problems ( 1 in 5 adolescents have one or more mental health problems). Adolescence is a habit forming age and many of them develop risk factors for future non-communicable diseases like diabetes, hypertension, and cancers, etc.

Developmentally too, adolescents are different than children and adults. On one hand they require supervised independence and on the other hand, they need privacy and 
confidentiality in health care. Thus, the needs of adolescents in health care delivery system varies. ${ }^{1}$

\section{Adolescence and their Needs}

The World Health Organization (WHO) defines "adolescents" as people aged 10-19 years; "youth" (also named "AYA" for "adolescents and young adults") as those aged 15 to 24 years; and "young people" as those aged 10-24 years. ${ }^{3}$

Adolescence is a crucial time in the life of every individual. There are a lot of physical, mental, emotional, psychological, hormonal and behavioral changes taking place that make impossible for an adolescent to understand what is happening with them. They are not considered as a child as physical changes takes place and not as adult because of their lack of wisdom. They are confused and irritated, hyperactive but do not know how to channelize this energy. Adolescence have different needs than adults.

They start to recognize demands of society and find ways to reconcile these with their own desires, motivations and a sense of self. In doing so, they strive to establish self-sufficiency by building tangible (e.g. skills, financial, educational credentials) and intangible (resilience, interpersonal skills) capacities to adapt to a society. It is also a period for young people to make commitments through the process of actively exploring and negotiating their identity. ${ }^{2}$

While everyone matures and goes through the changes in adolescence, this does not take place at the same time and in the same way for everyone. Some people mature early, others mature later. Also, all changes related to adolescence (physical, psycho-social and cognitive) do not take place at the same time. As a result, two adolescents of the same age may look very different. It is also possible that physical changes may take place early, but psycho-social changes take place later in the same individual. As a result, the adolescent may look very grown up, but may continue to think and behave like a child.

Often, adolescents feel inadequate because they look different from their peers. This could be because they are maturing too fast or slower than others. This difference often becomes the focus of teasing and ridicule among peers, leading to the experience of shame and fear. There are a lot of myths and beliefs attached to the growing up process, which need to be discussed. Adolescence is marked by changes that are not only physical but also psychological, social and emotional. ${ }^{3}$

\section{Adolescence and Health Needs}

Adolescence, the period between childhood and adulthood, is rightly viewed as a period of enormous change so their health care needs. Presently, there are 1.2 billion adolescents globally ${ }^{4}$ who are a valuable resource for their countries, ${ }^{5}$ but they are also at an increased risk of mortality and morbidity due to intentional and unintentional injuries, mental health problems, pregnancy-related complications and various life-threatening communicable diseases (such as Human Immunodeficiency Virus (HIV), Hepatitis B, etc.) .6,7

Decrease in high-risk behavior, mortality and morbidity and mortality and improvement in health status among adolescents will require a lot of effort and it's a life-long course, cannot be achieved in few days or months. But that does not mean that health care system should not start working on the same. Health care system should be well equipped to meet the unique demands of young adolescents. These services should be acceptable, efficient, equitable and safe as per the guidelines of WHO.

Trends of disease in Adolescent have changed a lot worldwide. Now adolescents are dealing more with mental health problems than infectious diseases. Sexual transmitted diseases and substance abuse, suicide, obesity are also on rise.

Health seeking behavior of adolescents, what are the barriers?

A study done by Kumar and et al8 showed that majority $(81 \%)$ of the adolescents reported some health problem and $(60 \%)$ of their problems were psychological and behavioral in nature. To resolve these problems boys consulted mainly friends/ peers (48\%) while girls consulted their mothers (63\%). Adolescents have greater counselling needs for psychosocial problems than for medical problems. Schoolbased adolescent health clinic was utilized more often than the dispensary-based clinic particularly for psychosocial problems. We can conclude that adolescents found it difficult to seek help from medical professionals and they approached their parents and peers.

Adolescents usually seek help for 1 . Their general physical health needs, 2. Specific developmental needs like school, vocation, employment, changes due to puberty and 3 . Personal psychosocial stressors like relationship problems, mental health, abuse etc.

They need health-related informational support, affiliative support, and emotional support, which includes close friends or family members, or professionals, who provide help for emotional needs or personal crises.

Help-seeking and having access to and using social supports are generally protective factors for many adolescent health and developmental outcomes. Having and using social supports is associated with lower rates of suicide, safer sexual behavior, lower rates of substance use, later sexual debut and lower rates of delinquency or perpetration of violence.

There can be various barriers which cause the non-utilization 
of the Health care facilities by the adolescents. They may be lack of faith, lack of confidentiality, lack of respect, judgmental attitude of the health care professionals, discrimination etc.

\section{Respectful Adolescent Care}

It is the right of every adolescent to get treated with respect and when it comes to health care, it becomes increasingly important to be polite, understanding non-judgmental on the part of health professionals so that adolescents feel comfortable and confident in seeking health care on time without any hesitation or delay.

Adolescents face many problems and along with problems if they want to approach health professionals, they face many barriers too. Services for adolescents exist but they are not updated and need significant improvement. WHO has suggested that health sector needs to transform its ways of dealing with adolescents. WHO has developed eight global standards to improve quality of health care services for adolescents.

WHO has clearly stated in standard 4 that there are certain competencies which all health care providers should demonstrate especially respectful care, confidentiality, privacy, non-discrimination etc. WHO has also emphasized on In-service training rather than Pre-service education, which will help in meeting the objectives.

The technical and attitudinal competency of healthcare providers is central to the implementation of all the standards. Therefore, competency-based educational programmes in adolescent health are necessary for implementation of the Global Standards for Quality HealthCare Services for Adolescents. Health care professionals should be familiar with the concept of Respectful Adolescent Care i.e. adolescents' rights to information, privacy, confidentiality, non-discrimination, nonjudgmental attitude and respect.

\section{Confidentiality: A Right of every Adolescent}

International guidelines recommend that it is right of every adolescent to participate and take decision regarding their illness and treatment. Every approach towards adolescent should be respectful. It has been reported that many times when the Adolescents approach the health care centers, they get remarks which hurt them and prevent them from getting the appropriate help.

In a study done to explore the factors impacting on access and acceptance of sexual and reproductive health services provided by adolescent-friendly health services in Nepal, results indicated that attention needs to be given to increase the capacity of health care providers to deliver services without imposing their own and socially sanctioned moral frameworks around adolescent sexual behavior. Such capacity building should include training that is experiential and emphasizes the importance of confidentiality and nonjudgmental attitudes. ${ }^{9}$

Facilities available for adolescents now in our health care system. Is it enough?

In India, Ministry of Health and Family welfare launched Rashtriya Kishor Swasthya Karyakram (RKSK) on January 7,2014 to ensure holistic development of adolescent. Rashtriya Kishor Swasthya Karyakram (RKSK) covers a large area like nutrition, Injuries, violence, non-communicable diseases, mental health and substance abuse. This programme mainly emphasized on health promotion of adolescents.

Keydrivers of the program are community-based interventions like, outreach by counselors; facility-based counselling; Social and Behavior Change Communication; and strengthening of Adolescent Friendly Health Clinics across levels of care. ${ }^{10}$

Adolescents are not being involved in decision making and to help them preventive, curative and counselling services are being provided at primary, secondary and tertiary levels during their clinic sessions.

\section{Respectful Adolescent Care in India}

Respectful Adolescent care is not provided to Adolescents by the health care providers because they do not have proper training. Also, there is paucity of material, money and manpower. Specially trained health care workers for Adolescent health services is the need of the hour. Sometimes, adolescents are not comfortable with the health care providers and scared of revealing personal issues.

To achieve wholesome adolescent health, we need to have a multidimensional respectful approach covering all the adolescent health problems with special emphasis on mental health, behavior change communication towards healthy lifestyle and positive social environment to acquire life skills. Adolescent friendly clinics need to be widely established throughout India and should achieve universal coverage. Empower and involve adolescents in decisions that affect them and facilitate them with every opportunity for developing into a successful adult. Offering such opportunities to the growing children gives them a chance to build a safe, happy, healthy and productive nation in the future. ${ }^{11}$

Adolescents often face the problems when approaching health as attitude is the main problem. Therefore, Health care professionals should be sensitive enough to understand the problems of the Adolescents and should have Objective and non-judgmental approach. In India, Respectful Adolescent Care is not practiced on a large scale and that's a point of concern. 


\section{Conclusion}

Adolescents are an important asset to any country and each and every country should invest in their health. If the youth especially the adolescents are happy, healthy and productive then the future of a country will be bright. Every individual is prone to commit mistakes and can be careless so are the adolescents. Therefore, adolescents should get guidance, health education and supervision and in case of any medical condition if they approach health care professionals, then they should be given Respectful Adolescent Care.

\section{Conflict of Interest: None}

\section{References}

1. Pemde PHK, Adolescent Friendly Health Clinics in India - Don't We Need Respectful Adolescent Health Care? The Indian Journal of Pediatrics 2019; 86(2): DOI: 10.1007/s12098-018-02843-x.

2. Adolescent patients and the clinical decision about their health Rev. paul. pediatr. vol.37 no.4 São Paulo Oct./Dec. 2019 Epub June 19, 2019; https:// doi.org/10.1590/1984-0462/;2019;37;4;00011.

3. Côté JE. Identity formation and self-development in adolescence. In R. M. Lerner \& L. Steinberg (Eds.), Handbook of adolescent psychology: Individual bases of adolescent development. 2009; 266-304). John Wiley \& Sons Inc. https:// doi.org/10.1002/9780470479193.adlpsy001010 Adolescent development - WHO ; www.who.int > topics > adolescence , development

4. UN Department of Economic and Social Affairs. United Nations; New York, NY: 2011 World population prospects: The 2010 revision. [Google Scholar].

5. Blum RW, Bastos FI, Kabiru CW. Adolescent health in the $21^{\text {st }}$ century. Lancet 2012; 379: 15671568. [PubMed] [Google Scholar].

6. Sawyer SM, Afifi RA, Bearinger LH. Adolescence: A foundation for future health. Lancet 2012; 379: 16301640. [PubMed] [Google Scholar].

7. Patton GC, Coffey C, Cappa C. Health of the world's adolescents: A synthesis of internationally comparable data. Lancet 2012; 379: 1665-1675. [PubMed] [Google Scholar].

8. Kumar R, Prinja S, Lakshmi PVM. Health care seeking behavior of adolescents: comparative study of two service delivery models. Indian J Pediatr 2008; 75(9): 895-899.

9. Pandey Pushpa Lata. Exploring the factors impacting on access and acceptance of sexual and reproductive health services provided by adolescent-friendly health services in Nepal, PLoS One 2019; 14(8): e0220855.

10. Adolescent Health (RKSK): National Health Mission; nhm.gov.in.
11. Sivagurunathan C, Umadevi R, Rama R, Gopalakrishnan S; Adolescent Health: Present Status and Its Related Programmes in India. Are We in the Right Direction?; J Clin Diagn Res 2015; 9(3). 